\title{
Optimizing Fourier filtering for digital holographic particle image velocimetry
}

\author{
Thomas Ooms $^{1}$, Wouter Koek ${ }^{2}$, Joseph Braat ${ }^{2}$ \\ and Jerry Westerweel ${ }^{1}$ \\ ${ }^{1}$ Laboratory for Aero- and Hydrodynamics, Faculty of Mechanical Engineering, \\ Delft University of Technology, Leeghwaterstraat 21, 2628 CA Delft, \\ The Netherlands \\ ${ }^{2}$ Optics Research Group, Faculty of Applied Sciences, Delft University of Technology, \\ Lorentzweg 12628 CJ Delft, The Netherlands \\ E-mail: t.a.ooms@wbmt.tudelft.nl
}

Received 16 August 2005, in final form 24 October 2005

Published 4 January 2006

Online at stacks.iop.org/MST/17/304

\begin{abstract}
In digital holographic particle image velocimetry, the particle image depth-of-focus and the inaccuracy of the measured particle position along the optical axis are relatively large in comparison to the characteristic transverse dimension of the reconstructed particle images. This is the result of a low optical numerical aperture (NA), which is limited by the relatively large pixel size of the CCD camera. Additionally, the anisotropic light scattering behaviour of the seeding particles further reduces the effective numerical aperture of the optical system and substantially increases the particle image depth-of-focus. Introducing an appropriate Fourier filter can significantly suppress this additional reduction of the NA. Experimental results illustrate that an improved Fourier filter reduces the particle image depth-of-focus. For the system described in this paper, this improvement is nearly a factor of 5 . Using the improved Fourier filter comes with an acceptable reduction of the hologram intensity, so an extended exposure time is needed to maintain the exposure level.
\end{abstract}

Keywords: digital holography, digital holographic particle image velocimetry, Fourier filtering, HPIV, DHPIV

\section{Introduction}

Digital holographic particle image velocimetry (DHPIV) makes it possible to determine the three-dimensional displacement field of small tracer particles carried by a fluid and uses a digital image sensor to record a hologram [1-4]. It provides many practical advantages by avoiding the time-consuming chemical processing of silver halide plates in conventional holographic particle image velocimetry. However, as a consequence of the relatively large CCD (or CMOS) pixel size (typically 5-10 $\mu \mathrm{m}$, or about ten times the wavelength of the illuminating light) the optical numerical aperture (NA) of a DHPIV set-up is limited. As a result, a point-like object such as a tracer particle with a typical size of a few tens of micrometres has a theoretical depth-of-focus (DOF) of typically more than $1 \mathrm{~mm}$. This implies a significant limitation of the spatial resolution along the optical axis of the system.

The maximum NA that follows from the finite CCD pixel size is referred to as the nominal NA. In addition, the anisotropic forward-scattering behaviour of the seeding particles makes the effective NA even smaller than the nominal NA, as most of the scattered light travels forward in a very narrow cone [5], as illustrated in figure 1. Although recording at $90^{\circ}$ side-scattering would largely avoid this, the extreme reduction of the angular light intensity of approximately a factor of $10^{12}$ for $30 \mu \mathrm{m}$ particles (figure 1) makes this an unfavourable solution.

Various approaches have focused on improving the spatial resolution along the optical axis. Stereoscopic holography can successfully remove the uncertainty along one axis by viewing from another (perpendicular) direction [1, 6, 7]. Pan and Meng 


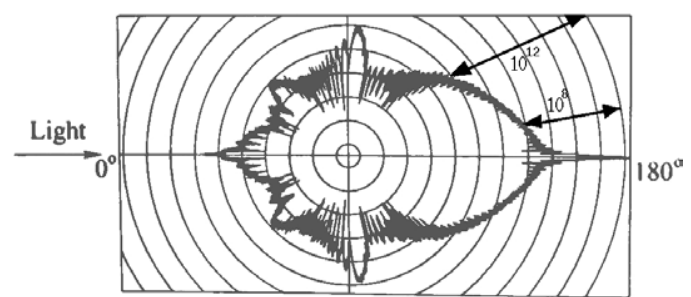

Figure 1. Scattered light intensity as a function of the scattering angle for a $30 \mu \mathrm{m}$ particle. The intensity scale is logarithmic and each circle represents a light intensity increase of a factor 100. (Source: M Raffel, C Wilert and J Kompenhans Particle Image Velocimetry, A Practical Guide [5]).

studied the use of the phase of the reconstructed light wave (only available in digital holography) to increase the accuracy of the reconstructed particle depth coordinate [2]. Although this work reports an impressive spatial resolution along the optical axis of approximately $10 \mu \mathrm{m}$, it cannot be applied successfully to transparent particles [2].

While the above methods aim at a general improvement of the spatial resolution along the optical axis, the following methods specifically aim to suppress the anisotropic scattering behaviour of the seeding particles, thereby attempting to increase the effective NA towards the nominal NA. Yang, Kostinski and Shaw [8] recently proposed numerical processing of the digital hologram to decouple particle size and particle position information. This method aims at the reduction of the particle image depth-of-focus by modifying a digital hologram in the frequency domain. However, this method requires foreknowledge of the particle size within 5\% [8]. Further, the scattered light intensity anisotropy of our particles is typically six orders of magnitude in the relevant range. Because the dynamic range of a typical CCD camera is only three orders of magnitude, we expect that the dynamic range and the noise level of the camera would limit the effectiveness of this method.

Alternatively, an appropriate optical filter can provide a solution for this problem without large requirements of the dynamic range of the camera. Previously, optical Fourier filters with a very small beam-stop were used to remove only the unscattered light while maintaining a high hologram exposure [6, 7]. Because the particle scattering profile is nearly unchanged, the anisotropic character of the object beam remains. This leads to a system with an effective NA that is significantly smaller than the nominal NA, as explained in section 2. Liu and Hussain [9] proposed an improved optical filter for a film-based (non-digital), off-axis holographic PIV set-up. Improvement of the depth-of-focus was investigated only for a single Fourier filter. In this paper, we build on their work by investigating various Fourier filters that have beam-stops with increasing diameter. The Fourier filters are specifically designed for application in forward-scattering digital holographic PIV. The effect of the different filters on the effective NA and the particle image depth-of-focus is investigated. We demonstrate that the optical performance is optimal with a Fourier filter with a beam-stop of intermediate size, and that this filter successfully increases the effective NA towards the nominal NA.

Section 2 contains a simple analysis of the relation between NA and particle image DOF for a digital in-line

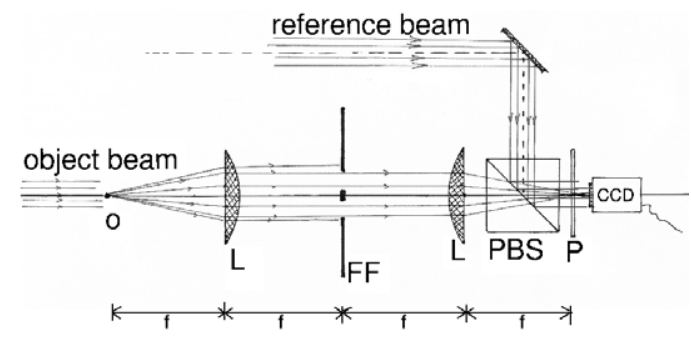

Figure 2. Optical configuration for recording a digital hologram. O: the recorded object; L: a plano-convex lens; FF: an optical Fourier filter; PBS: a polarizing beam splitter; P: a linear polarizer; $f$ : the focal length of the lenses.

holographic system, and explains how anisotropic scattering from particles affects the effective NA of the optical system. Section 3 supports section 2 with two numerical models. The holographic set-up and numerical reconstruction method that was used to study the effect of Fourier filtering is discussed in section 4 . The results and conclusions are given in sections 5 and 6 respectively.

\section{Analysis}

Most DHPIV systems [1-3] consist of an optical configuration with a single beam that is both the object beam and the reference beam. In this paper, we consider a modified system with spatially separated object and reference beams and a Fourier band pass filter, as shown in figure 2. This allows for filtering of the object beam and improvement of the optical performance of the system.

In a set-up with a plane-wave reference beam, the maximum nominal NA of the optical configuration depends on the pixel spacing of the CCD/CMOS sensor and the illumination wavelength. To achieve a successful hologram reconstruction, the Nyquist sampling criterion requires that the highest spatial frequency of the interference pattern projected on the sensor be less than $1 /(2 \Delta)$, where $\Delta$ is the pixel spacing. This implies that the angle between the propagation direction of any object light and the reference beam should be less than $\phi_{\max }$ and is given by

$$
\phi_{\max }=\arcsin (\lambda / 2 \Delta),
$$

where $\lambda$ is the illumination wavelength. If the reference beam is parallel to the optical axis, $\phi_{\max }$ represents the maximal angle between the object beam and the optical axis. For a typical DHPIV system $\phi_{\max }$ is a few hundredths of a radian, so the nominal NA $\left(\mathrm{NA}_{\text {nom }}\right)$ is equal to $\phi_{\max }$ in radians. An effective way to limit the range of propagation angles of the scattered object light is to include a circular ${ }^{3}$ aperture in the Fourier plane of the Fourier filter. The appropriate diameter $D$ of the aperture is given by

$$
D=2 f \sin \left(\phi_{\max }\right),
$$

where $f$ is the focal length of the Fourier filter lenses. The diameter $D$ is typically a few tens of millimetres with an $f$ of a few hundred millimetres. The diffraction limit $\delta$ (transverse dimension of a point-source image) of this optical system is

3 Analysing the square arrangement of the sensor pixels on the CCD chip with a two-dimensional Fourier transform indicates that a square-shaped aperture is needed for the most effective cut-off at the Nyquist (spatial) frequency. However, a circular aperture is applied to match the circular symmetry of particle scattering. 


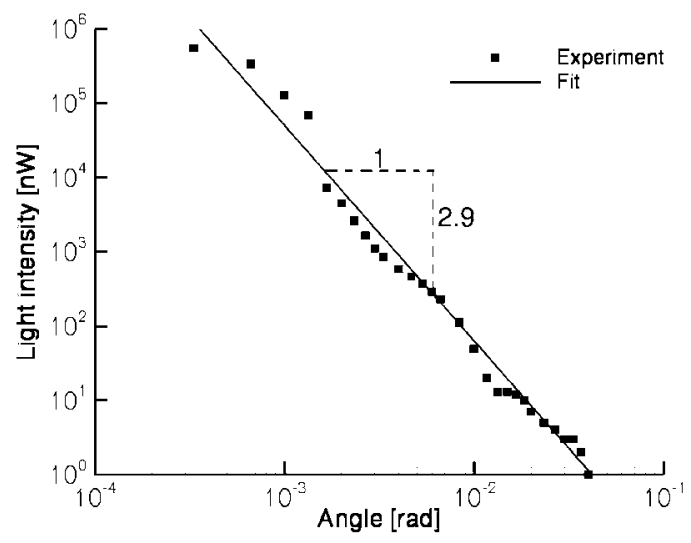

Figure 3. The measured light power as a function of the scattering angle for 40-63 $\mu \mathrm{m}$ glass particles (spherical) suspended in resin.

given by [10]

$$
\delta=1.22 \lambda / \mathrm{NA}_{\text {nom }},
$$

which is typically between 10 and $20 \mu \mathrm{m}$.

Additionally, the particle light scattering behaviour greatly influences the optical performance of the system. The intensity of the scattered light shows a very strong decay for increasing scattering angle. In the relevant range of scattering angles (0.00-0.04 rad), the scattered light intensity can decay several orders of magnitude. For example, using 40-63 $\mu \mathrm{m}$ particles and green $(532 \mathrm{~nm})$ light, the scattered light intensity decays approximately six orders of magnitude in the relevant angular range (section 4.1), as shown in figure 3. Due to the strong particle scattering anisotropy, the light rays that scatter from the object with a relatively large angle and that contribute most to the localization of the reconstructed particle image have a negligible contribution to the hologram. Therefore, the strong anisotropy in the scattering intensity leads to an increase of the particle image depth-of-focus. This increase in particle image DOF can be interpreted in terms of a reduced numerical aperture, which will be referred to as the effective NA $\left(\mathrm{NA}_{\mathrm{eff}}\right)$ of the optical system. (The particle image depthof-focus $\mathrm{DOF}_{\mathrm{p}}$ is defined as the full-width half-maximum (FWHM) of the intensity peak along a longitudinal line that crosses the centre of a particle image. This term is sometimes also referred to as the geometrical DOF and should not be confused with the conventional DOF, which refers to the longitudinal dimension of a diffraction-limited point-source image.)

When the particle diameter, $d_{p}$, is larger than the diffraction limit, the particle image depth-of-focus $\left(\mathrm{DOF}_{\mathrm{p}}\right)$ is approximately given by

$$
\mathrm{DOF}_{\mathrm{p}} \approx d_{p} / \mathrm{NA}_{\mathrm{eff}} .
$$

Because the effective NA is always smaller than the nominal $\mathrm{NA}$, the typical $\mathrm{DOF}_{\mathrm{p}}$ is larger than the $\mathrm{DOF}_{\mathrm{p}}$ in the ideal case when $\mathrm{NA}_{\text {eff }}=\mathrm{NA}_{\text {nom. }}$. Hence, the $\mathrm{DOF}_{\mathrm{p}}$ is often significantly larger that the $1 \mathrm{~mm}$ stated in section 1. Clearly, such a $\mathrm{DOF}_{\mathrm{p}}$ is much larger that the transverse dimension of typical seeding particles and leads to a poor spatial resolution along the optical axis.

To suppress the increase of the particle image DOF due to anisotropic particle scattering, an opaque disc with a diameter $C$ is placed on the optical axis in the Fourier plane of the Fourier
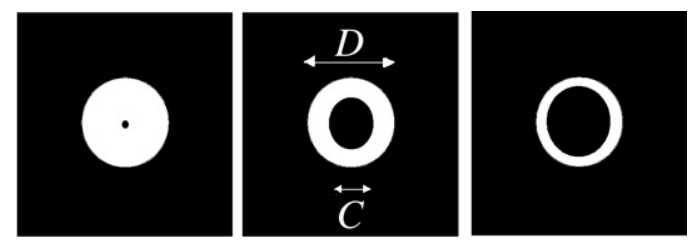

Figure 4. Three Fourier filters that were analysed; from left to right: the small-disc filter, the intermediate-disc filter and the large-disc filter. The diameter of the aperture is constant.

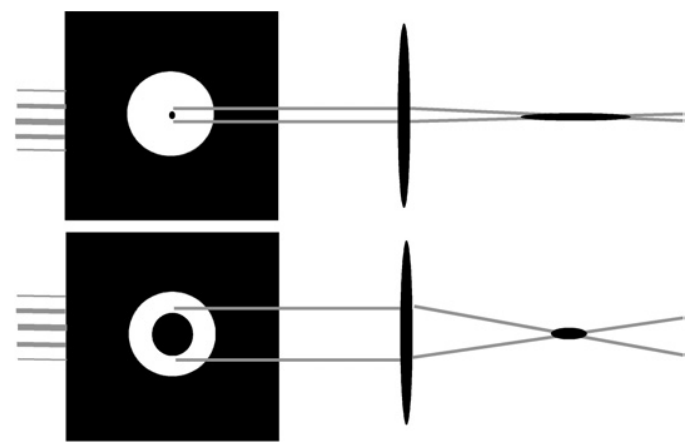

Figure 5. The small-disc filter and the intermediate-disc filter with the second Fourier lens and a real particle image. For clarity, the filter is rotated into the plane of the page. It is shown that the theory of geometrical optics predicts a smaller particle image DOF with the intermediate-disc filter versus the small-disc filter.

filter. The disc is concentric with respect to the outer aperture (figure 4). The filter factor $\gamma$ is introduced and defined as

$$
\gamma=(D-C) / C \text {. }
$$

To study the effect of the Fourier filter on the particle image depth-of-focus, three filters were tested: (1) a small-disc filter where $C \ll D$, or $\gamma \gg 1$; (2) an intermediate-disc filter where $C \approx \frac{1}{2} D$, or $\gamma \approx 1$; and (3) a large-disc filter where $C$ is only slightly smaller than $D$ (i.e., $C \approx D$ ), or $\gamma \ll 1$. The small-disc filter (with $C \ll D$ ) corresponds to a conventional beam-stop that is supposed to block only the non-scattered light. (The diameter of such a beam-stop should be at least 1-2 times the diffraction limit of the primary Fourier lens, which is typically a few micrometres; in most practical cases this requirement is amply fulfilled.)

Due to the strong particle scattering anisotropy, the majority of the light scatters from the object with a small angle and reaches the Fourier plane near the optical axis [10]. The light intensity decays rapidly with increasing distance from the optical axis. Therefore, most of the light transmitted by the spatial filter is centred closely around the inner opaque disc. Hence, relatively most of the light that passes the small-disc filter corresponds to a small scattering angle and the effective NA of the system remains considerably smaller than the available nominal NA. By increasing the diameter of the inner disc to an intermediate value, and being able to increase the CCD exposure time without overexposing, the light further from the optical axis will make a larger contribution to the hologram. This is expected to lead to an increased effective NA and decreased particle image DOF (4) as is shown schematically in figure 5. However, it is expected that the large-disc filter has such a narrow transmitting ring 
that the diffraction limit begins to influence the particle image DOF: if any opaque disc is placed inside the larger aperture, equation (3) is no longer a correct formulation of the diffraction limit [10]. Although for a small inner disc the effect of the inner disc on the diffraction limit is negligible, the inner disc of the large-disc filter significantly increases the diffraction limit of the optical system. Because the diffraction limit becomes larger than the particle diameter, the particle diameter $d_{p}$ in equation (4) should be replaced by the (larger) diffraction limit $\delta$, which implies that the further increase of $C$ leads to an increase of the particle image DOF. Summarizing, a typical DHPIV system with a Fourier filter, and with particle diameters in the range of tens of micrometres, is expected to have a minimal particle image DOF with an intermediate-disc filter. This is the result of a trade-off between an increasing effective NA and an increasing diffraction limit of the optical system for an increasing $C$. The exact size of the optimal intermediate disc depends on the specific experimental parameters. For example, the optimal diameter of the inner disc is closely related to the particle size. It is expected that if particle diameters are in the single-micrometre range, resulting in nearisotropic scattering, a filter with a smaller inner disc results in a minimal particle image depth-of-focus. Similarly, the optimal size of the inner disc of the Fourier filter is expected to be larger for bigger particles. The optimal diameter of the aperture of the Fourier filter is independent of the particle size. Therefore, the optimal $\gamma$ is larger for small particles and smaller for bigger particles. When focal lengths of the lenses vary, it is expected that the size of both the inner disc and the aperture in the Fourier plane should scale linearly, as described by equation (2). Hence, the optimal $\gamma$ would be independent of the focal length of the lenses. If the wavelength and CCD pixel size vary, the aperture diameter should, for such a low NA, scale proportional to the wavelength and inversely proportional to the CCD pixel size, as shown by equations (1) and (2). The optimal inner-disc diameter is expected to scale comparably, but not necessarily proportionally, to the diameter of the aperture. This corresponds to a possibly weak dependence of $\gamma$ on the wavelength and CCD pixel size.

The appropriate exposure time of the CCD camera depends on the size of the inner disc and the scattered light intensity as a function of the scattering angle. Increasing the inner-disc diameter, and so reducing $\gamma$, reduces the total amount of transmitted light and therefore implies a proportionally increased exposure time.

\section{Simple numerical model}

Two numerical models are used to investigate the effect of various Fourier filters on the longitudinal profile of a particle image.

The calculation consists of the following steps: light is numerically propagated from a particle, along the first ' $f$ ', distance, through the first lens, along the second ' $f$ ' distance, through the filter in the Fourier plane, along the third ' $f$ ', distance, through the second lens, and then to the real particle image. In both models, the particle is a point source on the optical axis that scatters isotropically. The Fresnel diffraction integral [10] is applied for propagation of light along the optical axis. Both lenses are modelled by a thin-lens approximation (quadratic exponential phase delay) [10]. The filter in the Fourier plane has a fully transmitting region, where the light amplitude is unchanged, and opaque regions, where the light amplitude is set to zero. To visualize the longitudinal profile of the particle image, propagation after the second lens is performed several times over a distance that ranges from ( $f-\Delta z)$ to $(f+\Delta z)$, where $f$ is the focal length of the lens and $\Delta z$ is half of the longitudinal range of interest. Throughout the system, the light field is modelled as a complex-amplitude scalar. Conversion to light intensity is the last step.

The first model illustrates the effect of a filter with a fixed aperture diameter $(D=24 \mathrm{~mm})$ and a varying inner-disc diameter $(C=1-20 \mathrm{~mm}$ ) (figure $6(a))$ on the longitudinal profile of the particle image. Figure $6(b)$ shows the increasing particle image DOF for an increasing $C$. In section 2 this increase is associated with the changing diffraction limit of the system. The increase of the particle image DOF is also shown in figure $6(c)$. The decreasing transmission for increasing $\mathrm{C}$ is also illustrated in figure $6(c)$. Because in this model, scattering is isotropic, transmission is proportional to $\left(D^{2}-C^{2}\right) / D^{2}$. Clearly, this model favours the use of a small inner disc to obtain a small particle image DOF and a high transmission. This would explain the frequent former application of small inner discs in HPIV set-ups [6, 7].

However, the strong light intensity anisotropy with scattering from realistic particles cannot be ignored. The anisotropy effect is included in the second model.

Although the anisotropy can be introduced by modelling Mie-scattering from a tracer particle, this approach is not followed because the extensive mathematics and physics of Mie-scattering theory is considered beyond the scope of this paper. Instead, the anisotropy is modelled by an adjustment in the Fourier filter. As described in section 2, the anisotropy leads to a concentration of the light just around the inner disc. For this reason, the anisotropy is here modelled by defining the Fourier filter as a fully transmitting ring with an inner diameter $C$ and an outer diameter $1.2 C$, where $C$ ranges from $1-20 \mathrm{~mm}$. The filter is opaque elsewhere. The power-law dependence of the realistic light intensity in the Fourier plane on the radius (section 4.1), and choosing the ring's outer diameter proportional to the inner diameter, makes this model identical to thresholding the realistic light intensity in the Fourier plane. For the proportionality value of 1.2 (chosen for practical reasons) thresholding occurs at (section 4.1) $\left(1.2^{-2.9}=\right.$ ) 0.59 times the intensity at the edge of the inner disc. In other words, in this model, Fourier plane positions $(x, y)$ where the realistic light intensity is larger than the threshold $(C<r<$ $1.2 C$ ), the modelled light amplitude is constant and finite, and where the realistic light intensity is smaller than the threshold ( $r<C, r>1.2 C$ ), the modelled light amplitude is zero (figure 6(d)). Figure 6(e) shows that the second model predicts a decreasing particle image DOF for an increasing inner-disc diameter (opposite to the trend in figure $6(b) !)$. This result is also shown in figure $6(f)$. The second model clearly favours the use of a large inner disc for a minimal particle image DOF.

Because the particle image DOF curve in figure 6(c) rises sharply at high $C$-values and this curve in figure $6(f)$ rises sharply at low $C$-values, these two results could intuitively be combined to predict that, in practice, a minimal particle 


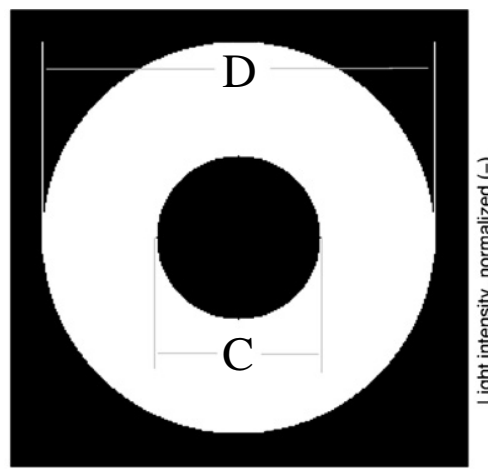

(a)

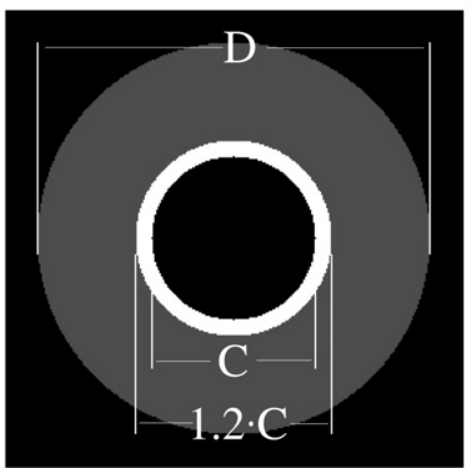

(d)

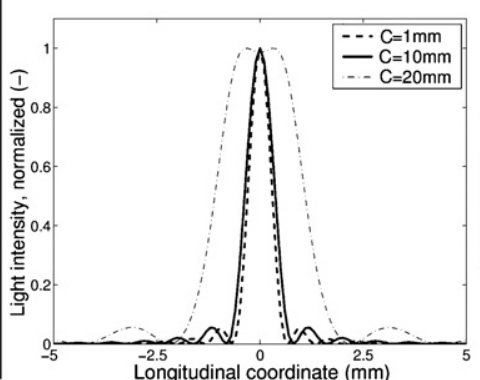

(b)

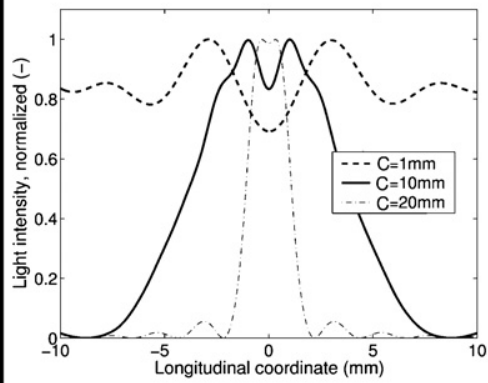

(e)

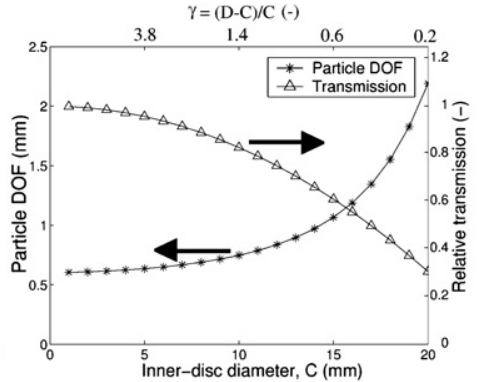

(c)

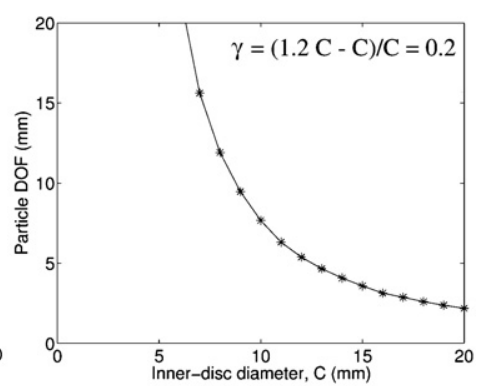

(f)

Figure 6. Two simple numerical models that show the effect of various Fourier filters on the longitudinal profile of the particle image. Figures $(a)-(c)$ correspond to the first model with isotropic scattering. Figures $(d)-(f)$ correspond to the second model, where the realistic anisotropic scattering is modelled as a constant light intensity on a narrow ring around the inner disc. The inner diameter of the ring is $C$, the outer diameter is $1.2 C$. With $C=20 \mathrm{~mm}$, both models are identical. The scales of the horizontal axes in $(b)$ and $(e)$ are different for optimal visualization of the various curves.

DOF can be obtained with a Fourier filter with an intermediate inner-disc diameter. This supports ${ }^{4}$ the content of section 2 .

\section{Experimental procedure}

\subsection{Digital hologram recording}

The three different Fourier filter configurations were tested in an in-line digital holographic optical system as shown in figure 2. The illumination source of the set-up is a diodepumped continuous-wave $150 \mathrm{~mW} \mathrm{Nd:YAG} \mathrm{laser} \mathrm{(Coherent,}$ Compass 315M-150) with a light wavelength of $532 \mathrm{~nm}$. The laser beam is expanded, collimated and then split into separate object and reference beams. The object is a resinfilled tank with stationary $40-63 \mu \mathrm{m}$ tracer particle (Sphericel) at a density of approximately $10^{3}$ particles per $\mathrm{cm}^{3}$. This object with stationary particles allows for full control of the position of the particles and hence for calibration measurements on the system. The depth of the tank along the optical axis is $20 \mathrm{~mm}$.

The hologram is recorded by a lens-less digital CCD camera (PCO Sensicam 690LL) with $1376 \times 1040$ pixels and a pixel spacing of $6.45 \mu \mathrm{m}$. According to equation (1) the

4 Despite the fair predictions of these models, we would still not recommend the use of a numerical model for the prediction of the optimal inner-disc diameter. Finding an exact optimal value of $C$ requires an improved model with Mie-scattering simulations, which implies a considerable computational effort. An experimental approach, however, requires the optimization of only one parameter, namely the inner-disc diameter, $C$. Production and testing of a few filters typically takes only a few days, making it a far more practical approach. maximum angle between the reference beam and object light should be less than $0.041 \mathrm{rad}$. The reference beam and the object beam are combined and propagate towards the CCD camera along the same axis. This on-axis set-up is appropriate for digital holography, because, for a plane-wave reference beam, fringes have a maximum size.

The Fourier filter consists of a spatial filter and two identical positive plano-convex lenses with a $300 \mathrm{~mm}$ focal length and $50 \mathrm{~mm}$ diameter. The filter and the lenses are all separated by the lens focal length. Equation (2) shows that a focal length of $300 \mathrm{~mm}$ leads to a practically sized spatial filter with an aperture diameter of $24 \mathrm{~mm}$.

The Fourier-filtered object light and the reference beam are combined by a beam splitter and travel through a linear polarizer. This polarizer ensures uniform polarization at the CCD chip and suppresses other states of polarization introduced when light scatters from the object. If the object light and reference beam at the CCD are not polarized uniformly, it is expected that hologram fringe contrast is negatively affected.

An important aspect is the positioning of the camera. For our configuration, the camera was positioned in a region between 1 and $3 \mathrm{~cm}$ behind the real image of the particles that is created by the lenses of the Fourier filter. If the CCD is positioned closer to the real image, the virtual image in the reconstruction approaches the brightness of the real image, making it difficult to distinguish the real image from the virtual image and preventing a successful reconstruction of the particle locations. If the CCD is positioned too close to 
the real image, it is also expected that the signal-to-noise ratio of the hologram reconstruction would be negatively affected, because a reconstructed particle image would be derived from a smaller area on the sensor (i.e., fewer hologram pixels). This makes the reconstruction more sensitive to CCD camera noise. On the other hand, if the CCD camera is positioned too far from the real image, only a part of a diverging out-of-focus particle image falls onto the CCD chip. This would negatively affect the particle image depth-of-focus, because it is expected that the entire circular out-of-focus image should be recorded to allow for an optimal particle reconstruction.

\subsection{Numerical reconstruction}

Rayleigh-Sommerfeld diffraction theory [10] is applied to obtain the light amplitude in 200 parallel reconstruction planes. The hologram image, $I_{h}$, is regarded as a grating and the light amplitude in a reconstruction plane, $U_{\mathrm{z}}$, is calculated by convoluting the hologram intensity with a kernel $K_{\mathrm{z}}(x, y)$, representing the light wave emitted by a point source [10]. This is a well-described method for obtaining an image in a parallel reconstruction plane [11],

$$
U_{\mathrm{z}}(x, y)=I_{h}(x, y) * K_{\mathrm{z}}(x, y),
$$

with

$$
K_{\mathrm{z}}(x, y)=\frac{1}{\mathrm{i} \lambda} \frac{\exp \left(\mathrm{i} k \sqrt{x^{2}+y^{2}+z^{2}}\right)}{\sqrt{x^{2}+y^{2}+z^{2}}},
$$

where $*$ represents a two-dimensional (2D) convolution, $\mathrm{i}$ is the imaginary unit, $\lambda$ is the wavelength, $k$ is the wavenumber, $x$ and $y$ are the transverse coordinates, and $z$ is the distance from the CCD chip to a parallel reconstruction plane. The cosine term in the Raleigh-Sommerfeld solution can be neglected for this low-NA set-up. Equation (6) does not use the Fresnel approximation because the use of this approximation is possibly not justified for all $z$-values. The calculation time of the hologram reconstruction with or without application of the Fresnel approximation is identical.

The convolution is performed with $2 \mathrm{D}$ fast Fourier transform (FFT) algorithms on a personal computer (CPU: AMD $2.5 \mathrm{GHz}$; OS: Linux Red Hat) using Matlab 6.5. A selection of $1024 \times 1024$ pixels is cropped from the hologram to optimize the computing speed for the $2 \mathrm{D}$ FFTs. In the Fourier transform of the hologram, the spatial frequencies larger than $(D / 2 \cdot \lambda \cdot f)$, or $75 \mathrm{~mm}^{-1}$ in our case, should be zero due to the presence of the aperture in the Fourier plane of the recording set-up. The spatial frequencies smaller than $(C / 2 \cdot \lambda \cdot f)$ should also be zero, due to the presence of the opaque disc in the Fourier plane. Pixel values outside the resulting 'doughnut' in the numerical Fourier plane that, against expectation, are not equal to zero, are considered to be noise and numerically set to zero.

The kernel $K_{\mathrm{z}}(x, y)$ has only finite (non-zero) values on a disc where the angle between the propagation direction of the light and the optical axis is sufficiently small to avoid aliasing in the hologram, i.e., smaller than 0.041 radians. The obtained Fourier transform of the hologram and the Fourier transform of the kernel are multiplied and the product is inversely Fourier transformed. The complex-amplitude image is multiplied by its own conjugate to obtain the light intensity in the reconstruction plane. This process is repeated for all

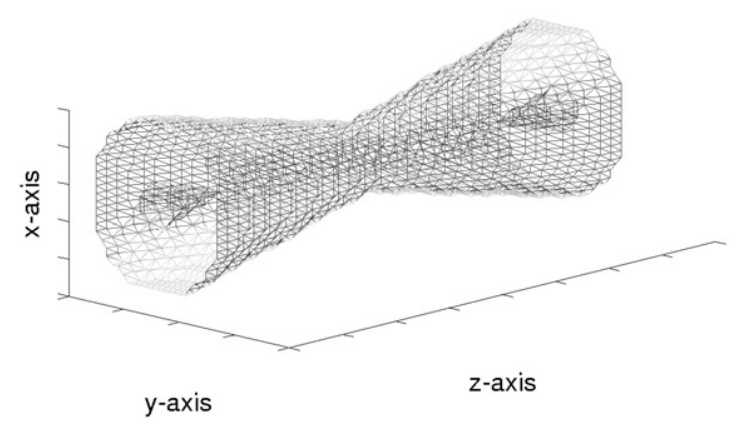

Figure 7. A modelled three-dimensional light intensity profile of a real particle image near its focus. The scale of the transverse $(x, y)$ axis does not correspond to the scale of the longitudinal (z) axis.

reconstruction planes, each separated by $100 \mu \mathrm{m}$, to obtain a volume of $6.6 \times 6.6 \times 20 \mathrm{~mm}^{3}$ containing $1024 \times 1024 \times$ 200 volume elements or voxels.

The following procedure is applied for the particle detection: a reference volume is defined numerically that contains an estimated three-dimensional (3D) light intensity pattern of a real image of a single particle. As shown in figure 7, the reference volume consists of a cone along the optical axis of $13 \times 13 \times 19$ voxels, corresponding to a volume of $84 \times 84 \times$ $1900 \mu \mathrm{m}^{3}$. This synthetic reference volume is correlated with the previously determined total volume, resulting in a volume with several correlation maxima. This volume is scanned for local maxima that are higher than one-half of the maximum correlation value in the volume. These points are assumed to represent actual particle positions and the 3D coordinates of all particles are stored in an array. The analysis typically takes $1 \mathrm{~h}$ per reconstructed volume (using 1.5 Gbyte physical memory).

\section{Optimizing Fourier filtering}

\subsection{Measurement of the scattered light intensity profile}

The optimal design of the Fourier filter depends strongly on the anisotropic scattering behaviour of the particles. To study the scattering behaviour of our particles in more detail, the light intensity is measured as a function of the scattering angle in an intermediate experiment.

Using the set-up as described in section 4, the light intensity in the Fourier plane was measured directly by means of a power meter with a light sensitive area of about $1 \mathrm{~mm}^{2}$. The measured light intensity pattern as a function of a transverse coordinate in the Fourier plane can be interpreted as the scattering light intensity as a function of the scattering angle at the object [10]. The measured light intensity is plotted as a function of the scattering angle in figure 3. The graph clearly shows the decrease of light intensity with increasing scattering angle. In a double logarithmic graph, the data points are approximately distributed along a straight line. This suggests a power-law relation between the light intensity and the scattering angle,

$$
I=A / \phi^{B}
$$

where $I$ is the light intensity (in $\mathrm{nW} \mathrm{mm}^{-2}$ ), $\phi$ is the scattering angle, and $A$ and $B$ are numerical coefficients. A fit of the data in figure 3 to the power law in equation (7) yields 


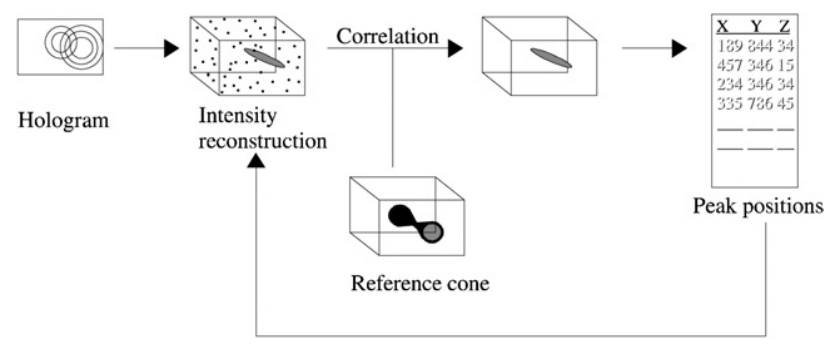

Figure 8. The steps that lead to the profiles of figure 9.

$A=10^{-4} \mathrm{nW} \mathrm{rad}{ }^{B} \mathrm{~mm}^{-2}$ and $B=2.9$. The value of $A$ depends on the particular laser power and the optical configuration. The value of $B$ is close to 3 , which would be the expected exponent for light diffracted by a small circular aperture when ignoring fine-structured variations [10].

\subsection{Measuring the effect of different Fourier filters}

Three different filters were applied in the holographic system described in the previous section, i.e., with $C=1 \mathrm{~mm}$ (smalldisc filter, $\gamma=23$ ), $10 \mathrm{~mm}$ (intermediate-disc filter, $\gamma=1.4$ ), and $20 \mathrm{~mm}$ (large-disc filter, $\gamma=0.2$ ). (The diameter $D$ of the anti-aliasing aperture is $24 \mathrm{~mm}$; see the previous section.) The relative area of transmission of the filter is $\left(D^{2}-\right.$ $\left.C^{2}\right) / D^{2}$, and equals $99.8 \%, 82.6 \%$ and $30.6 \%$ for the respective filters. Because the appropriate CCD exposure time should be inversely proportional to the surface-integrated light intensity at the filter, the CCD exposure times would, with the respective filters and in the case of isotropic particle scattering, relate as $1.00: 1.21: 3.26$. However, because particle scattering is strongly anisotropic, these ratios of the CCD exposure time are expected to be significantly larger.

Following the reconstruction and particle detection procedure described in the previous section, the positions of about 100 particles per hologram were determined. The light intensity along a longitudinal line, which intersects a particle centre, is determined for all particles, and this was repeated for each of the Fourier filters. The longitudinal intensity profiles are averaged over all particles for each Fourier filter. The procedure is shown schematically in figure 8 , and the result is shown in figure 9. The graph in figure 9 shows that the $\mathrm{DOF}_{\mathrm{p}}$ (defined as the FWHM of the longitudinal profile) for the $1 \mathrm{~mm}, 10 \mathrm{~mm}$ and $20 \mathrm{~mm}$ discs are 5.34, 1.08 and $2.99 \mathrm{~mm}$ respectively. As expected, for the three filters the $10 \mathrm{~mm}$ intermediate-disc filter yields results with the smallest $\mathrm{DOF}_{\mathrm{p}}$. Note that the $\mathrm{DOF}_{\mathrm{p}}$ for the $1 \mathrm{~mm}$ small-disc filter (i.e., the one that would correspond to a conventional DHPIV setup) spans a significant part of the full depth of the measurement domain. The $\mathrm{DOF}_{\mathrm{p}}$ for the intermediate-disc $10 \mathrm{~mm}$ filter is 4.9 times smaller than the $\mathrm{DOF}_{\mathrm{p}}$ corresponding to the smalldisc $1 \mathrm{~mm}$ filter, and 2.8 times smaller than the $\mathrm{DOF}_{\mathrm{p}}$ corresponding to the large-disc $20 \mathrm{~mm}$ filter. Figure 10 shows a slice of the intensity profile of a single particle image for each Fourier filter, and confirms the results shown in figure 9. It also shows that the transverse particle image size increases notably for the $20 \mathrm{~mm}$ filter as a result of the increased diffraction limit for the large-disc filter (as explained in section 2). The CCD exposure times are $10 \mu \mathrm{s}, 500 \mu \mathrm{s}$ and $2750 \mu \mathrm{s}$ for the respective filters. This confirms that the appropriate CCD

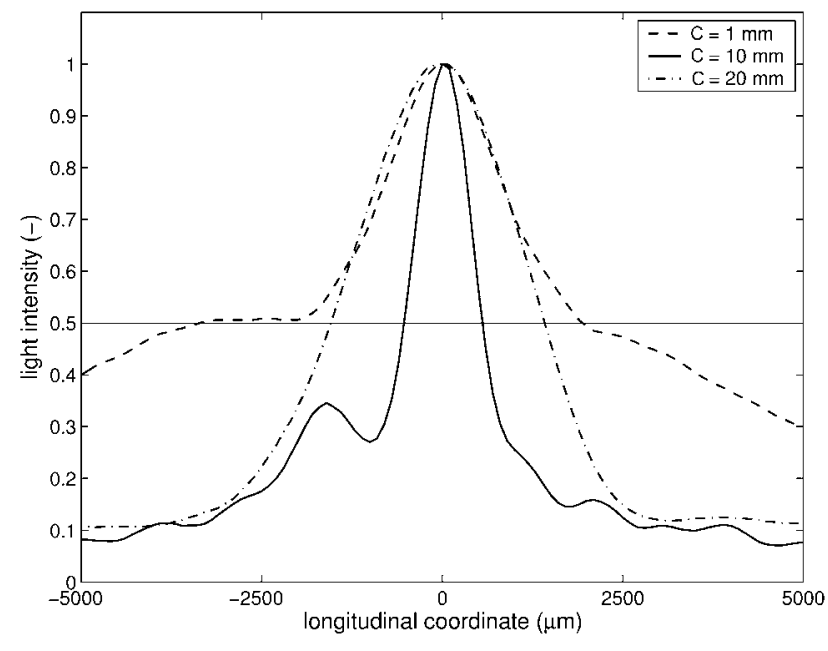

Figure 9. Experimental result: the average light intensity as a function of the longitudinal coordinate in holographic reconstructions of about 100 seeding particles for the three Fourier filters.

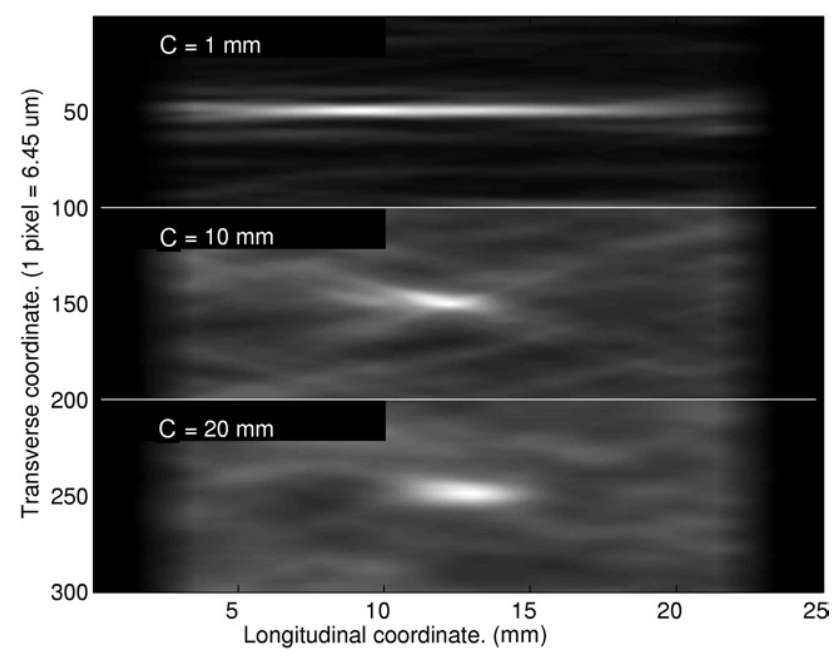

Figure 10. Three reconstructions of one particle, recorded with the three different Fourier filters. An increase of $C$ from 1 to $10 \mathrm{~mm}$ leads to a significant reduction of the particle image DOF. The increased transverse dimension of the particle image is clearly visible for $C=20 \mathrm{~mm}$.

exposure time ratios are significantly larger than the ratios corresponding to isotropic scattering, namely $1: 50: 275$. These ratios are theoretically confirmed within a factor 3 when integrating the light intensity of equation (7) over the Fourier plane and taking the reciprocal values to obtain the exposure times.

These experimental results are summarized in table 1: the effective NA is calculated by dividing the smallest particle diameter, i.e., $40 \mu \mathrm{m}$, by the measured FWHM particle image depth-of-focus (4). The ratio of the effective NA and nominal NA is based on a nominal NA of 0.041 , and equals $18 \%, 90 \%$ and $32 \%$ for the small-disc filter, intermediate-disc filter and large-disc filter respectively.

The use of an enlarged $(10 \mathrm{~mm})$ inner disc implies a reduction of the amount of transmitted light that reaches the CCD sensor. If increasing the exposure time to compensate for the reduction of light intensity is not possible (for example, due 
Optimizing Fourier filtering for digital holographic particle image velocimetry

Table 1. Summary of measurement results. Calculations are described in section 5 .

\begin{tabular}{lllllr}
\hline Filter: $C(\mathrm{~mm})$ & $\gamma(=(D-C) / C)$ & Depth-of-focus $(\mathrm{mm})$ & $\mathrm{NA}_{\text {eff }}(-)$ & $\mathrm{NA}_{\text {eff }} / \mathrm{NA}_{\text {nom }}(\%)$ & Exposure time $(\mu \mathrm{s})$ \\
\hline 1 & 23 & 5.34 & $7.5 \times 10^{-3}$ & 18 & 10 \\
10 & 1.4 & 1.08 & $3.7 \times 10^{-2}$ & 90 & 500 \\
20 & 0.2 & 2.99 & $1.3 \times 10^{-2}$ & 32 & 2750 \\
\hline
\end{tabular}

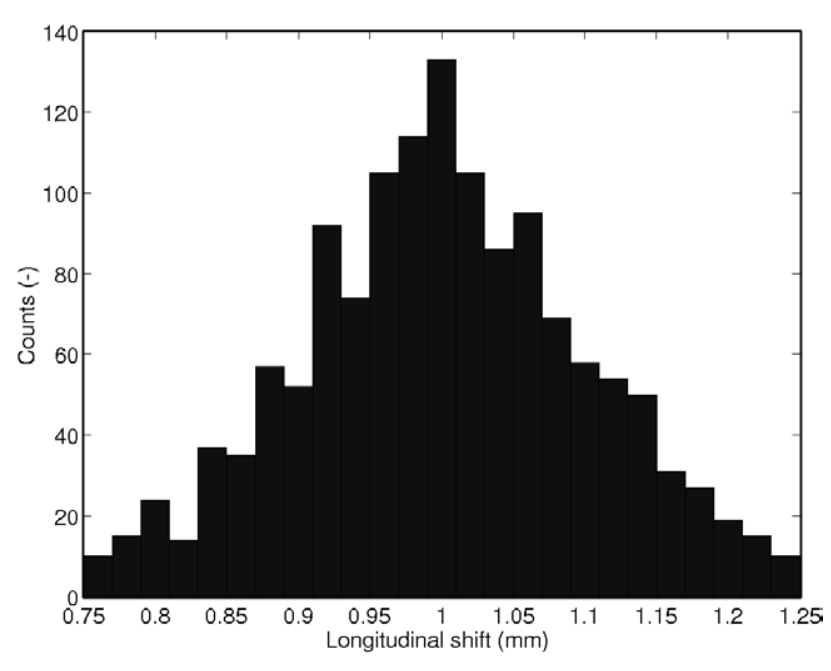

Figure 11. Histogram of longitudinal shifts of reconstructed particles. All particles were shifted exactly $1 \mathrm{~mm}$.

to particle motion), it can be compensated by an increase in the amount of light supplied by the light source. Although roughly 50 times less light reaches the CCD camera with the $10 \mathrm{~mm}$ filter in comparison to the $1 \mathrm{~mm}$ filter, available lasers have sufficient power to compensate for this. For example, with an exposure time of $500 \mu \mathrm{s}$, the continuous-wave $150 \mathrm{~mW}$ laser delivers $75 \mu \mathrm{J}$ to the system. A pulsed laser could easily deliver $75 \mathrm{~mJ}$ per pulse.

Further, the effect of omission of the outer aperture from the Fourier filter is studied. Although it may be expected that the outer aperture is redundant due to the low light intensity away from the optical axis, experimental results indicated that the particle image longitudinal resolution slightly deteriorated after removing the outer aperture.

Because the effectiveness of a DHPIV set-up largely depends on the ability of the system to accurately reconstruct particle positions, and because the spatial resolution along the optical axis is relatively poor, the measured longitudinal particle position accuracy is further analysed.

With the set-up described in section 4 and the $10 \mathrm{~mm}$ Fourier filter, a holographic recording is made of the described tank with fixed particles at a certain position and of the same tank after it was moved exactly $1 \mathrm{~mm}$ along the optical axis. Two reconstructed volumes are derived from the two holograms and particle positions are determined as described in section 4. Then, for each particle, the longitudinal subpixel coordinate is obtained by performing a seven-point leastsquares Gaussian fit. The measured longitudinal particle position is a direct result of one of the fit parameters. Then, by subtracting the longitudinal coordinate of each particle before and after the $1 \mathrm{~mm}$ shift, a set of displacements is obtained, which should be distributed around $1 \mathrm{~mm}$. These data are shown as a histogram in figure 11. The result shows that the centre of the histogram is located at $1 \mathrm{~mm}$, which means that there is no bias in the measured displacement and that there is no undesired longitudinal scaling factor present in the experimental set-up and numerical reconstruction procedure. Secondly, the approximate fullwidth half-maximum (FWHM) of the histogram is $200 \mu \mathrm{m}$. This value is about one order of magnitude smaller than the FWHM of the average longitudinal intensity profile (solid line in figure 9), which agrees with a rule of thumb that it should be possible to experimentally determine the position of a Gaussian peak with an accuracy that is one order of magnitude better than the typical width of the peak [12-14].

The transverse particle coordinates are simply derived from the integer pixel point of local maximum light intensity. The accuracy of the transverse particle coordinate is therefore nearly two orders of magnitude better than the longitudinal particle coordinate, so no effort is currently made for its improvement.

\section{Conclusions}

The particle image depth-of-focus and effective numerical aperture of a DHPIV system are primarily determined by the anisotropic scattering of light by the tracer particles. It is demonstrated that the effects due to the anisotropic scattering can be reduced by means of an appropriate Fourier filter that blocks the scattered light near the optical axis of the optical system. Three filter configurations were tested with filter factors $\gamma=23, \gamma=1.4, \gamma=0.2$ which correspond to $1 \mathrm{~mm}$, $10 \mathrm{~mm}$ and $20 \mathrm{~mm}$ diameter opaque discs respectively. For the $10 \mathrm{~mm}$ filter, the particle image depth-of-focus is 4.9 times smaller than for the $1 \mathrm{~mm}$ filter and 2.8 times smaller than for the $20 \mathrm{~mm}$ filter. These results agree with the theoretical prediction of geometrical optics that the $10 \mathrm{~mm}$ filter has a smaller particle image DOF than the $1 \mathrm{~mm}$ filter. These results also agree with the expectation that, for the $20 \mathrm{~mm}$ filter, the particle image DOF has again increased due to a significant increase of the transverse size of the particle image as a result of an increase in the diffraction limit of the optical system. These results are also in agreement with the two numerical models in section 3. It is shown in section 5 that the reduction of light that reaches the CCD sensor with the intermediate-disc filter can, next to adjustment of the CCD exposure time, be easily compensated by the power of available lasers. It is further demonstrated that the uncertainty for the measured position of a single particle along the optical axis is about $200 \mu \mathrm{m}$. This uncertainty for a digital HPIV system is close to the reported inaccuracies of optical holographic PIV systems using high-resolution films or plates [5]. These conclusions indicate that the optical performance of the system is optimal for the intermediate-disc filter.

Because previous publications describe holographic recording set-ups with small high-pass filters $[6,7]$ or without 
any Fourier filtering at all [1-3], it is expected that there may be room for improvement in the effective NA and $\mathrm{DOF}_{\mathrm{p}}$ of both conventional and digital holographic PIV systems.

\section{Acknowledgments}

We would like to thank the Dutch FOM foundation for their financial support, Koh Ikeda for the useful discussions and Wilco Tax for his technical support.

\section{References}

[1] Malkiel E, Sheng J, Katz J and Strickler J R 2003 The 3-dimensional flow field generated by a feeding calanoid copepod measured using digital holography J. Exp. Biol. 206 3657-66

[2] Pan G and Meng H 2003 Digital holography of particle fields: reconstruction using complex amplitude Appl. Opt. $\mathbf{4 2}$ $827-33$

[3] Ikeda K, Okamoto K and Murata S 2003 Development of dynamic digital holographic particle velocimetry 5th Int. Symp. on Particle Image Velocimetry (Busan, Korea, 22-24 Sept.) ed S J Lee et al p 3103

[4] Hinsch K D 2002 Holographic particle image velocimetry Meas. Sci. Technol. 13 R61-72
[5] Raffel M, Wilert C and Kompenhans J 1998 Particle Image Velocimetry A Practical Guide ed R J Adrian et al (Berlin: Springer)

[6] Sheng J, Malkiel E and Katz J 2003 Single beam two-views holographic particle image velocimetry Appl. Opt. 42 235-50

[7] Zhang J, Tao B and Katz J 1997 Turbulent flow measurement in a square duct with hybrid holographic PIV Exp. Fluids 23 373-81

[8] Yang W, Kostinski A B and Shaw R A 2005 Depth-of-focus reduction for digital in-line holography of particle fields Opt. Lett. 30 1303-5

[9] Liu F and Hussain F 1998 Holographic particle velocimeter using forward scattering with filtering Opt. Lett. 23 132-34

[10] Goodman J W 1996 Introduction to Fourier Optics ed S W Director et al (Singapore: McGraw-Hill)

[11] Schnars U and Jüptner W 1994 Direct recording of holograms by a CCD target and numerical reconstruction Appl. Opt. 33 179-81

[12] Adrian R J 1991 Particle-imaging techniques for experimental fluid mechanics Ann. Rev. Fluid Mech. 23 261-304

[13] Westerweel J 2000 Theoretical analysis of the measurement precision in particle image velocimetry Exp. Fluids $29 \mathrm{~S} 3-12$

[14] Prasad A K, Adrian R J, Landreth C C and Offutt P W 1992 Effect of resolution on the speed and accuracy of particle image velocimetry interrogation Exp. Fluids 13 105-16 